\title{
Investigation of TCF7L2, LEP and LEPR polymorphisms with esophageal squamous cell carcinomas
}

\author{
Hao Qiu ${ }^{1, *}$, Xunting Lin ${ }^{2,}{ }^{,}$, Weifeng Tang ${ }^{3}$, Chao Liu ${ }^{3}$, Yu Chen ${ }^{4}$, Hao Ding ${ }^{5}$, Mingqiang \\ Kang ${ }^{6,7,8}$ and Shuchen Chen ${ }^{6}$ \\ ${ }^{1}$ Department of Immunology, School of Medicine, Jiangsu University, Zhenjiang, Jiangsu Province, China \\ ${ }^{2}$ Department of Gastroenterology, Zhongshan Hospital Xiamen University, Xiamen, Fujian Province, China \\ ${ }^{3}$ Department of Cardiothoracic Surgery, Affiliated People's Hospital of Jiangsu University, Zhenjiang, Jiangsu Province, China \\ ${ }^{4}$ Department of Medical Oncology, Fujian Cancer Hospital, Fujian Medical University Cancer Hospital, Fuzhou, Fujian Province, \\ China \\ ${ }^{5}$ Department of Respiratory Disease, Affiliated People's Hospital of Jiangsu University, Zhenjiang, Jiangsu Province, China \\ ${ }^{6}$ Department of Thoracic Surgery, Fujian Medical University Union Hospital, Fuzhou, Fujian Province, China \\ ${ }^{7}$ Key Laboratory of Ministry of Education for Gastrointestinal Cancer, Fujian Medical University, Fuzhou, Fujian Province, \\ China \\ ${ }^{8}$ Fujian Key Laboratory of Tumor Microbiology, Fujian Medical University, Fuzhou, Fujian Province, China \\ *These authors have contributed equally to this work
}

Correspondence to: Shuchen Chen, email: cscdoctor@163.com

Keywords: TCF7L2; LEP; LEPR; polymorphism; ESCC

Received: June 05, $2017 \quad$ Accepted: August 26, $2017 \quad$ Published: November 17, 2017

Copyright: Qiu et al. This is an open-access article distributed under the terms of the Creative Commons Attribution License 3.0 (CC BY 3.0), which permits unrestricted use, distribution, and reproduction in any medium, provided the original author and source are credited.

\section{ABSTRACT}

Single nucleotide polymorphisms (SNPs) in energy metabolism related gene may be key agents in the development of human malignancies. In this study, we aimed to examine the association of transcription factor 7-like 2, Leptin (LEP) and LEP receptor (LEPR) polymorphisms with esophageal squamous cell carcinoma (ESCC). A total of 507 ESCC cases and 1,496 controls were enrolled. We found that LEPR rs6588147 AA genotype was associated with ESCC risk (AA vs. GG/GA: adjusted $O R=1.90,95 \% C I=1.00-3.61, P=0.049)$. In the stratified analyses, $L E P R$ rs6588147 G >A polymorphism increased the risk of ESCC ( $<63$ years subgroup: AA vs. GG: adjusted $O R=2.58,95 \% C I=1.00-6.62, P=0.049$ and $A A$ vs. $G A / G G:$ adjusted $O R=2.71,95 \% C I=1.06-6.91, P=0.038$; male subgroup: $A A$ vs. $G G$ : adjusted $O R=2.19$, 95\%CI $=1.02-4.67, P=0.044$ and $A A$ vs. $G A / G G$ : adjusted $O R=2.26,95 \% C I=1.06-$ 4.80, $P=0.035$ ). However, LEP rs7799039 A $>G$ decreased the risk of ESCC ( $\geq 63$ years subgroup: GG vs. AA: adjusted $O R=0.47,95 \% C I=0.23-0.95, P=0.035$ and $G G$ vs. $A A /$ AG: adjusted $O R=0.48,95 \% C I=0.24-0.96, P=0.038 ; B M I \geq 24 \mathrm{~kg} / \mathrm{m}^{2}$ subgroup: $A G$ vs. $A A$ : adjusted $O R=0.66,95 \% C I=0.45-0.99, P=0.044)$. In addition, $L E P R$ rs 1137101 G>A polymorphism decreased ESCC risk in some subgroups (ever smoking subgroup: GA vs. GG: adjusted $O R=0.66,95 \% C I=0.44-1.00, P=0.049$; ever drinking subgroup: GA vs. GG: adjusted $O R=0.54,95 \% C I=0.31-0.95, P=0.031$ and $G A / A A$ vs. $G$ : adjusted $O R=0.54,95 \% C I=0.31-0.93, P=0.027)$. Our findings suggest that $L E P R$ rs6588147 G>A polymorphism is associated with the increased risk of ESCC; however, LEP rs7799039 A > G and LEPR rs1137101 G>A polymorphisms may be protective factors for ESCC. 


\section{INTRODUCTION}

In China, esophageal cancer (EC) is the fourth most commonly diagnosed cancer in males and the fifth in females, with an estimated 477,900 new patients and 375,000 related deaths occurring in 2015 [1]. Esophageal squamous cell carcinoma (ESCC) is the main form of EC in China and Eastern Asia. The contributing risk factors for ESCC are not fully known, but are thought to involve low intake of vegetables and fruits, poor nutritional status, smoking and eating and/or drinking at high temperatures. However, these primarily identified risk factors could not account for all the etiology of ESCC. Nowadays, there are convincing evidences that obesity increases the susceptibility of many malignancies, including EC, postmenopausal breast cancer, endometrial cancer, colorectal cancer, pancreatic cancer and liver cancer [2]. A recent study indicated that preoperative metabolic syndrome might be an effective predictor of ESCC mortality [3]. These accumulating evidences suggested that obesity and diabetes related gene might play vital roles in the development of EC.

The transcription factor 7-like 2 (TCF7L2) gene maps to the long arm of chromosome 10q25.3. TCF7L2 belongs to the high mobility group-box (HMGB) family [4] and is a versatile transcription factor. The TCF7L2 protein regulates $\mathrm{Wnt} / \beta$-catenin signaling pathway [5], therefore it plays important roles in the development and growth of various cells $[6,7]$. Ishiguro et al. reported that TCF7L2 expression was associated with a poor prognosis of ESCC [8]. A previous study suggested that TCF7L2 rs7903146 locus might exert its enhancer function by interacting with HMGB1 [9]. TCF7L2 single-nucleotide polymorphisms (SNPs) are proposed susceptibility factors for the development of cancer. Previous studies indicated that TCF7L2 rs7903146 (C/T) polymorphism might influence the risk of breast cancer $[10,11]$. TCF7L2 rs290481 T>C polymorphism located on near the 3' end of this gene. Ling et al. reported that this SNP was associated with hepatocellular carcinoma susceptibility with marginal significance [12]. However, the association between these $T C F 7 L 2$ SNPs and ESCC risk was not explored.

The Leptin (LEP) gene maps to chromosome 7q31.3. LEP is secreted by white adipose tissue and has been identified to be involved in endocrinologic metabolism [13]. It is thought that LEP may regulate the activation and serum levels of insulin. Thus, LEP may involve in the etiology of obesity [14], type 2 diabetes (T2DM) [15] and pathophysiology of carcinoma [16, 17]. LEP receptor (LEPR, also known as CD295) is a single transmembrane protein in human and distributes in various tissues [18]. LEP combines to LEPR and exerts its important roles in the development of metabolic disorders and malignancies. Several studies demonstrated that the elevated LEP levels might affect the onset and progression of many malignancies [19-22]. Thus, LEP and LEPR may be correlated with the development of ESCC.
Results of meta-analyses found that both rs7799039 $\mathrm{A}>\mathrm{G}$ and $\mathrm{rs} 2167270 \mathrm{G}>\mathrm{A}$ polymorphisms in $L E P$ gene might influence the risk of cancer [23-25]. In addition, a case-control study found that $L E P$ rs $2167270 \mathrm{G}>\mathrm{A}$ was associated with the risk of esophageal adenocarcinoma [26]. There are several explanations for the function of these two LEP polymorphisms. It is suggested that rs7799039 $\mathrm{A}>\mathrm{G}$ polymorphism in the upstream region of LEP gene can affect leptin expression, possibly at the transcriptional level, thereby altering adipose secretion levels of the hormone [27]. Additionally, LEP rs 2167270 $\mathrm{G}>\mathrm{A}$ is a $5^{\prime}$-utr SNP and may play regulatory roles in translation and stability of mRNA. LEPR rs $1137100 \mathrm{G}>\mathrm{A}$, rs1137101 G>A polymorphisms are missense SNPs and may alter the structure and the function of LEPR protein. Doecke et al. found $L E P R$ rs1137100 G>A, rs1137101 $\mathrm{G}>\mathrm{A}$ polymorphisms influence the risk of esophageal adenocarcinoma in Caucasians [26]. LEPR rs6588147 $\mathrm{G}>\mathrm{A}$ polymorphism locates on the intron region of LEPR gene. Slattery et al. found that LEPR rs6588147 $\mathrm{G}>\mathrm{A}$ polymorphism affected risk of colon cancer among men [28]. However, the association between LEPR rs1137100 G>A, rs1137101 $\mathrm{G}>\mathrm{A}$ and $\mathrm{rs} 6588147 \mathrm{G}>\mathrm{A}$ polymorphisms and ESCC risk remains unknown in Asians.

In this case-control study, we aimed to examine the potential association of TCF7L2, LEP and LEPR polymorphisms with the risk of ESCC in Eastern Chinese Han populations. The TCF7L2 rs7903146 C > T, rs290481 $\mathrm{T}>\mathrm{C}, L E P$ rs7799039 $\mathrm{A}>\mathrm{G}, \mathrm{rs} 2167270 \mathrm{G}>\mathrm{A}$ and $L E P R$ rs1137100 $\mathrm{G}>\mathrm{A}$, rs1137101 $\mathrm{G}>\mathrm{A}$ and $\mathrm{rs} 6588147 \mathrm{G}>\mathrm{A}$ polymorphisms were genotyped by SNPscan genotyping assays in 507 ESCC cases and 1,496 non-cancer controls.

\section{RESULTS}

\section{Baseline characteristics}

There were 2,003 participants in the present casecontrol study including 507 ESCC patients ( 377 males and 130 females) and 1,496 non-cancer controls (1,084 males and 412 females). The age and sex were well matched in two groups $(\mathrm{P}=0.994, \mathrm{P}=0.406$, respectively, Table 1$)$. The mean \pm SD of weight and body mass index (BMI) was significantly higher in controls compared with ESCC patients $(P<0.05)$. However, the mean $\pm \mathrm{SD}$ of height was not significant $(P>0.05)$. The proportion of smoking and drinking was significantly higher in ESCC patients compared with controls $(P<0.05)$. Locus information of TCF7L2, LEP and LEPR polymorphisms is listed in Table 2. The genotyping success rates for TCF7L2 rs7903146C $>\mathrm{T}$, rs290481 $\mathrm{T}>\mathrm{C}, L E P$ rs7799039 $\mathrm{A}>\mathrm{G}$, rs2167270 $\mathrm{G}>\mathrm{A}$ and $L E P R$ rs1137100 $\mathrm{G}>\mathrm{A}$, rs 1137101 $\mathrm{G}>\mathrm{A}$ and $\mathrm{rs} 6588147 \mathrm{G}>\mathrm{A}$ SNPs were $99.50 \%, 99.45 \%$, $99.50 \%, 99.40 \%, 99.50 \%, 99.50 \%$ and $99.50 \%$, respectively. Minor allele frequency (MAF) in controls is listed in Table 2, which is very similar to the data of 
Table 1: Distribution of selected demographic variables and risk factors in ESCC cases and controls

\begin{tabular}{|c|c|c|c|c|c|}
\hline \multirow{2}{*}{ Variable } & \multicolumn{2}{|l|}{ Cases $(n=507)$} & \multicolumn{2}{|c|}{ Controls $(n=1,496)$} & \multirow{2}{*}{$P^{\text {a }}$} \\
\hline & $\mathbf{n}$ & $\%$ & $\mathbf{n}$ & $\%$ & \\
\hline Age (years) & $62.77( \pm 8.01)$ & & $62.77( \pm 8.84)$ & & 0.994 \\
\hline Age (years) & & & & & 0.225 \\
\hline$<63$ & 271 & 53.45 & 753 & 50.33 & \\
\hline$\geq 63$ & 236 & 46.55 & 743 & 49.67 & \\
\hline Sex & & & & & 0.406 \\
\hline Male & 377 & 74.36 & 1,084 & 72.46 & \\
\hline Female & 130 & 25.64 & 412 & 27.54 & \\
\hline Tobacco use & & & & & $<0.001$ \\
\hline Never & 247 & 48.72 & 1,090 & 72.86 & \\
\hline Ever & 260 & 51.28 & 406 & 27.14 & \\
\hline Alcohol use & & & & & $<0.001$ \\
\hline Never & 341 & 67.26 & 1,329 & 88.84 & \\
\hline Ever & 166 & 32.74 & 167 & 11.16 & \\
\hline Height $(\mathrm{cm})$ & $166.0( \pm 7.29)$ & & $166.1( \pm 7.08)$ & & 0.743 \\
\hline Weight (kg) & $61.54( \pm 9.83)$ & & $66.11( \pm 9.92)$ & & $<0.001$ \\
\hline BMI $\left(\mathrm{kg} / \mathrm{m}^{2}\right)$ & $22.27( \pm 2.90)$ & & $23.91( \pm 3.03)$ & & $<0.001$ \\
\hline BMI $\left(\mathrm{kg} / \mathrm{m}^{2}\right)$ & & & & & $<0.001$ \\
\hline$<24$ & 370 & & 779 & & \\
\hline$\geq 24$ & 137 & & 717 & & \\
\hline
\end{tabular}

a Two-sided $\chi^{2}$ test and student $\mathrm{t}$ test; Bold values are statistically significant $(P<0.05)$. BMI: body mass index.

Chinese population. In addition, the distributions of the TCF7L2 rs7903146C $>$ T, rs290481 T $>$ C, LEP rs7799039 $\mathrm{A}>\mathrm{G}, \quad \mathrm{rs} 2167270 \mathrm{G}>\mathrm{A}$ and $L E P R \quad$ rs1137100 $\mathrm{G}>\mathrm{A}$, rs1137101 $\mathrm{G}>\mathrm{A}$ and $\mathrm{rs} 6588147 \mathrm{G}>\mathrm{A}$ genotypes in controls conform to Hardy-Weinberg equilibrium (HWE).

\section{Association of TCF7L2 rs7903146C $>$ T, rs290481 $T>C$, LEP rs7799039 A $>G$, rs2167270 G $>A$ and LEPR rs1137100 G>A, rs1137101 $G>A$ and rs6588147 G $>$ A polymorphisms with ESCC risk}

The genotype distributions of TCF7L2 rs7903146C $>\mathrm{T}$, rs290481 $\mathrm{T}>\mathrm{C}, L E P$ rs7799039 $\mathrm{A}>\mathrm{G}$, rs2167270 $\mathrm{G}>\mathrm{A}$ and $L E P R$ rs1137100 $\mathrm{G}>\mathrm{A}$, rs1137101 $\mathrm{G}>\mathrm{A}$ and rs6588147 $\mathrm{G}>\mathrm{A}$ polymorphisms are listed in Table 3. In the analysis of $L E P R \operatorname{rs} 6588147 \mathrm{G}>\mathrm{A}$ polymorphism, we found significant differences in the distribution of the rs6588147 AA genotype compared with the rs6588147 GG genotype and rs6588147 AA genotype compared with the rs6588147 GA/GG genotypes between 507 ESCC cases and 1,496 controls [AA vs. GG: crude odds ratio $(\mathrm{OR})=1.87,95 \%$ confidence interval $(\mathrm{CI})=$
1.02-3.43, $P=0.042$ and AA vs. GG/GA: crude OR $=$ $1.93,95 \% \mathrm{CI}=1.06-3.53, P=0.031$ (Table 3 )]. Results of multivariate linear regression analysis indicated that $L E P R$ rs6588147 $\mathrm{G}>\mathrm{A}$ polymorphism increased the risk of ESCC. When the LEPR rs6588147 GG/GA genotypes were used as the reference group, the LEPR rs6588147 AA genotype was associated with the increased risk of ESCC [AA vs. GG/GA: adjusted OR $=1.90,95 \% \mathrm{CI}=$ 1.00-3.61, $P=0.049$ (Table 3)]. However, we found that TCF7L2 rs7903146C $>$ T, rs290481 T $>$ C, LEP rs7799039 $\mathrm{A}>\mathrm{G}, \quad \mathrm{rs} 2167270 \mathrm{G}>\mathrm{A}$ and $L E P R \quad$ rs1137100 $\mathrm{G}>\mathrm{A}$, rs $1137101 \mathrm{G}>\mathrm{A}$ polymorphisms were not associated with the development of overall ESCC (Table 3).

Association of TCF7L2 rs7903146C $>$ T, rs290481 $\mathrm{T}>\mathrm{C}$, LEP rs7799039 A>G, rs2167270 $\mathrm{G}>\mathrm{A}$ and LEPR rs1137100 G>A, rs1137101 $G>A$ and rs6588147 $\mathrm{G}>\mathrm{A}$ polymorphisms with ESCC risk in Different Stratification Groups

Table 4 shows the genotype frequencies of LEP rs7799039 $\mathrm{A}>\mathrm{G}$ polymorphism in the subgroup analyses. 
Table 2: Primary information for $T C F 7 L 2$ rs7903146C $>$ T, rs290481 T $>C, L E P$ rs7799039 A $>$ G, rs2167270 G $>A$ and $L E P R$ rs1137100 $\mathrm{G}>\mathrm{A}$, rs1137101 $\mathrm{G}>\mathrm{A}$ and $\mathrm{rs6588147} \mathrm{G}>\mathrm{A}$ polymorphisms

\begin{tabular}{|c|c|c|c|c|c|c|c|c|}
\hline $\begin{array}{l}\text { Genotyped } \\
\text { SNPs }\end{array}$ & Chromosome & $\begin{array}{c}\text { Chr Pos } \\
\text { (NCBI } \\
\text { Build 37) }\end{array}$ & Region & $\begin{array}{c}\text { MAFa for } \\
\text { Chinese in } \\
\text { database }\end{array}$ & $\begin{array}{c}\text { MAF } \\
\text { in our } \\
\text { controls }(n \\
=1,496)\end{array}$ & $\begin{array}{c}P \text { value } \\
\text { for } H_{W} \text { b } \\
\text { test in our } \\
\text { controls }\end{array}$ & $\begin{array}{c}\text { Genotyping } \\
\text { method }\end{array}$ & $\begin{array}{c}\text { Genotyping } \\
\text { value }(\%)\end{array}$ \\
\hline $\begin{array}{l}T C F 7 L 2 \\
\text { rs7903146 } \\
\mathrm{C}>\mathrm{T}\end{array}$ & 10 & 114758349 & Intron 4 & 0.026 & 0.031 & 0.733 & SNPscan & 99.50 \\
\hline $\begin{array}{l}\text { TCF7L2 } \\
\text { rs290481 } \\
\mathrm{T}>\mathrm{C}\end{array}$ & 10 & 114923825 & Intron 13 & 0.405 & 0.387 & 0.097 & SNPscan & 99.45 \\
\hline $\begin{array}{l}L E P \\
\text { rs7799039 } \\
\mathrm{A}>\mathrm{G}\end{array}$ & 7 & 127878783 & Promoter & 0.201 & 0.266 & 0.543 & SNPscan & 99.50 \\
\hline $\begin{array}{l}L E P \\
\text { rs2167270 } \\
\mathrm{G}>\mathrm{A}\end{array}$ & 7 & 127881349 & 5' UTR & 0.175 & 0.222 & 0.324 & SNPscan & 99.40 \\
\hline $\begin{array}{l}L E P R \\
\text { rs } 1137100 \\
\mathrm{G}>\mathrm{A}\end{array}$ & 1 & 66036441 & Exon 4 & 0.169 & 0.160 & 0.316 & SNPscan & 99.50 \\
\hline $\begin{array}{l}L E P R \\
\text { rs1137101 } \\
\mathrm{G}>\mathrm{A}\end{array}$ & 1 & 66058513 & Exon 6 & 0.111 & 0.122 & 0.763 & SNPscan & 99.50 \\
\hline $\begin{array}{l}L E P R \\
\text { rs6588147 } \\
\mathrm{G}>\mathrm{A}\end{array}$ & 1 & 65935494 & Intron 2 & 0.150 & 0.150 & 0.260 & SNPscan & 99.50 \\
\hline
\end{tabular}

${ }^{a}$ MAF: minor allele frequency.

${ }^{\mathrm{b}}$ HWE: Hardy-Weinberg equilibrium.

In $\geq 63$ years subgroup, after adjustment for gender, smoking status, BMI and alcohol use, the LEP rs7799039 GG genotype decreased ESCC risk compared with the LEP rs7799039 AA genotype genotype or $L E P$ rs 7799039 AA/ AG [GG vs. AA: adjusted OR $=0.47,95 \%$ CI $0.23-0.95$, $P=0.035$ and $\mathrm{GG}$ vs. $\mathrm{AA} / \mathrm{AG}$ : adjusted $\mathrm{OR}=0.48,95 \%$ $\mathrm{CI}=0.24-0.96, P=0.038$ (Table 4)]. In BMI $\geq 24 \mathrm{~kg} /$ $\mathrm{m}^{2}$ subgroup, after adjustment for age, gender, smoking status and alcohol use, we found that $L E P$ rs $7799039 \mathrm{AG}$ genotype decreased the risk of ESCC [AG vs. AA: adjusted $\mathrm{OR}=0.66,95 \%$ CI $0.45-0.99, P=0.044$ (Table 4)].

The genotype frequencies of $L E P R$ rs1137101 G>A polymorphism in the subgroup analyses are showed in Table 5. In ever smoking subgroup, after adjustment for gender, age, BMI and alcohol use, the LEPR rs $1137101 \mathrm{GA}$ genotype was associated with the decreased risk of ESCC [GA vs. GG: adjusted $\mathrm{OR}=0.66,95 \%$ CI $0.44-1.00, P=$ 0.049 (Table 5)]. In ever drinking subgroup, after adjustment for gender, smoking status, BMI and age, we found that LEPR rs1137101 GA and GA/AA genotypes decreased the risk of ESCC [GA vs. GG: adjusted OR $=0.54,95 \% \mathrm{CI}$
$0.31-0.95, P=0.031$ and $\mathrm{GA} / \mathrm{AA}$ vs. GG: adjusted $\mathrm{OR}=$ $0.54,95 \%$ CI $0.31-0.93, P=0.027$ (Table 5)].

Table 6 shows the genotype frequencies of $L E P R$ rs6588147 G $>$ A polymorphism in the subgroup analyses. In $<63$ years subgroup, after adjustment for gender, smoking status, BMI and alcohol use, the LEPR rs6588147 AA genotype increased ESCC risk compared with the LEPR rs6588147 GG and GA/GG genotypes [AA vs. GG: adjusted $\mathrm{OR}=2.58,95 \%$ CI $1.00-6.62, P=0.049$ and AA vs. GA/GG: adjusted OR $=2.71,95 \%$ CI $1.06-6.91$, $P=0.038$ (Table 6)]. In male subgroup, after adjustment for age, smoking status, BMI and alcohol use, the LEPR rs6588147 AA genotype was associated with the increased risk of ESCC [AA vs. GG: adjusted OR $=2.19,95 \% \mathrm{CI}$ $1.02-4.67, P=0.044$ and AA vs. GA/GG: adjusted $\mathrm{OR}=$ $2.26,95 \%$ CI 1.06-4.80, $P=0.035$ (Table 6)]. However, in ever drinking subgroup, after adjustment for age, gender, smoking status and BMI, the LEPR rs6588147 GA genotype decreased the risk of ESCC [GA vs. GG: adjusted $\mathrm{OR}=0.54,95 \%$ CI $0.31-0.92, P=0.024$ (Table 6)]. 
Table 3: Logistic regression analyses of association between $T C F 7 L 2$ rs7903146C $>T$, rs290481 $T>C, L E P$ rs7799039 $\mathrm{A}>\mathrm{G}, \mathrm{rs2167270} \mathrm{G}>\mathrm{A}$ and $L E P R$ rs1137100 $\mathrm{G}>\mathrm{A}, \mathrm{rs1137101} \mathrm{G}>\mathrm{A}$ and $\mathrm{rs6588147} \mathrm{G}>\mathrm{A}$ polymorphisms and risk of ESCC

\begin{tabular}{|c|c|c|c|c|c|c|c|c|}
\hline \multirow{2}{*}{ Genotype } & \multicolumn{2}{|c|}{ ESCC cases $(n=507)$} & \multicolumn{2}{|c|}{ Controls $(n=1,496)$} & \multirow{2}{*}{$\begin{array}{c}\text { Crude OR } \\
(95 \% \mathrm{CI})\end{array}$} & \multirow{2}{*}{$P$} & \multirow{2}{*}{$\begin{array}{c}\text { Adjusted OR a } \\
(95 \% \mathrm{CI})\end{array}$} & \multirow{2}{*}{$P$} \\
\hline & $\mathbf{n}$ & $\%$ & $\mathrm{n}$ & $\%$ & & & & \\
\hline \multicolumn{9}{|c|}{ TCF7L2 rs7903146C $>\mathrm{T}$} \\
\hline $\mathrm{CC}$ & 475 & 94.25 & 1,399 & 93.96 & 1.00 & & & \\
\hline $\mathrm{CT}$ & 29 & 5.75 & 89 & 5.98 & $\begin{array}{l}0.96(0.62- \\
1.48)\end{array}$ & 0.847 & $1.03(0.65-1.62)$ & 0.908 \\
\hline TT & 0 & 0 & 1 & 0.07 & - & - & - & - \\
\hline $\mathrm{CT}+\mathrm{TT}$ & 29 & 5.75 & 90 & 6.04 & $\begin{array}{c}0.95(0.62- \\
1.46)\end{array}$ & 0.814 & $1.01(0.64-1.60)$ & 0.954 \\
\hline $\mathrm{CC}+\mathrm{CT}$ & 504 & 100.00 & 1488 & 99.93 & 1.00 & & 1.00 & \\
\hline TT & 0 & 0 & 1 & 0.07 & - & - & - & - \\
\hline $\mathrm{T}$ allele & 29 & 2.88 & 91 & 3.06 & & & & \\
\hline \multicolumn{9}{|c|}{ TCF7L2 rs290481 $\mathrm{T}>\mathrm{C}$} \\
\hline TT & 195 & 38.77 & 575 & 38.62 & 1.00 & & & \\
\hline $\mathrm{TC}$ & 228 & 45.33 & 676 & 45.40 & $\begin{array}{l}0.99(0.79- \\
1.23)\end{array}$ & 0.903 & $0.96(0.76-1.22)$ & 0.748 \\
\hline $\mathrm{CC}$ & 80 & 15.90 & 238 & 15.98 & $\begin{array}{l}0.98(0.73- \\
1.33)\end{array}$ & 0.911 & $0.99(0.71-1.36)$ & 0.927 \\
\hline $\mathrm{TC}+\mathrm{CC}$ & 308 & 61.23 & 914 & 61.38 & $\begin{array}{l}0.99(0.81- \\
1.22)\end{array}$ & 0.952 & $0.98(0.78-1.22)$ & 0.830 \\
\hline $\mathrm{TT}+\mathrm{TC}$ & 423 & 84.10 & 1,251 & 84.02 & 1.00 & & 1.00 & \\
\hline $\mathrm{CC}$ & 80 & 15.90 & 238 & 15.98 & $\begin{array}{l}0.99(0.75- \\
1.31)\end{array}$ & 0.967 & $1.01(0.75-1.36)$ & 0.949 \\
\hline $\mathrm{C}$ allele & 388 & 38.57 & 1,152 & 38.68 & & & & \\
\hline \multicolumn{9}{|c|}{$L E P$ rs $7799039 \mathrm{~A}>\mathrm{G}$} \\
\hline AA & 291 & 57.74 & 797 & 53.53 & 1.00 & & 1.00 & \\
\hline AG & 184 & 36.51 & 591 & 39.69 & $\begin{array}{l}0.85(0.69- \\
1.05)\end{array}$ & 0.138 & $0.85(0.67-1.06)$ & 0.144 \\
\hline GG & 29 & 5.75 & 101 & 6.78 & $\begin{array}{l}0.79(0.51- \\
1.21)\end{array}$ & 0.275 & $0.73(0.46-1.17)$ & 0.191 \\
\hline $\mathrm{AG}+\mathrm{GG}$ & 213 & 42.26 & 692 & 46.47 & $\begin{array}{l}0.84(0.69- \\
1.03)\end{array}$ & 0.101 & $0.83(0.67-1.03)$ & 0.091 \\
\hline $\mathrm{AA}+\mathrm{AG}$ & 475 & 94.25 & 1,388 & 93.22 & 1.00 & & 1.00 & \\
\hline GG & 29 & 5.75 & 101 & 6.78 & $\begin{array}{l}0.84(0.55- \\
1.28)\end{array}$ & 0.419 & $0.79(0.50-1.24)$ & 0.300 \\
\hline $\mathrm{G}$ allele & 242 & 24.01 & 793 & 26.63 & & & & \\
\hline \multicolumn{9}{|c|}{$L E P$ rs $2167270 \mathrm{G}>\mathrm{A}$} \\
\hline GG & 318 & 63.35 & 894 & 60.04 & 1.00 & & 1.00 & \\
\hline GA & 165 & 32.87 & 528 & 35.46 & $\begin{array}{l}0.87(0.70- \\
1.08)\end{array}$ & 0.213 & $0.87(0.69-1.09)$ & 0.220 \\
\hline AA & 19 & 3.78 & 67 & 4.50 & $\begin{array}{c}0.79(0.47- \\
1.34)\end{array}$ & 0.382 & $0.81(0.47-1.42)$ & 0.469 \\
\hline
\end{tabular}




\begin{tabular}{|c|c|c|c|c|c|c|c|c|}
\hline \multirow{2}{*}{ Genotype } & \multicolumn{2}{|c|}{ ESCC $\operatorname{cases}(n=507)$} & \multicolumn{2}{|c|}{ Controls $(n=1,496)$} & \multirow{2}{*}{$\begin{array}{c}\text { Crude OR } \\
(95 \% \mathrm{CI})\end{array}$} & \multirow{2}{*}{$P$} & \multirow{2}{*}{$\begin{array}{l}\text { Adjusted OR a } \\
(95 \% C I)\end{array}$} & \multirow{2}{*}{$\boldsymbol{P}$} \\
\hline & $\mathbf{n}$ & $\%$ & $\mathbf{n}$ & $\%$ & & & & \\
\hline $\mathrm{GA}+\mathrm{AA}$ & 184 & 36.65 & 595 & 39.96 & $\begin{array}{l}0.87(0.71- \\
1.07)\end{array}$ & 0.190 & $0.86(0.69-1.08)$ & 0.198 \\
\hline $\mathrm{GG}+\mathrm{GA}$ & 483 & 96.22 & 1,422 & 95.50 & 1.00 & & 1.00 & \\
\hline AA & 19 & 3.78 & 67 & 4.50 & $\begin{array}{c}0.84(0.50- \\
1.40)\end{array}$ & 0.496 & $0.86(0.49-1.50)$ & 0.591 \\
\hline A allele & 203 & 20.22 & 662 & 22.23 & & & & \\
\hline \multicolumn{9}{|c|}{$L E P R$ rs $1137100 \mathrm{G}>\mathrm{A}$} \\
\hline GG & 342 & 67.86 & 1,045 & 70.18 & 1.00 & & 1.00 & \\
\hline GA & 147 & 29.17 & 411 & 27.60 & $\begin{array}{c}1.09(0.87- \\
1.37)\end{array}$ & 0.448 & $1.08(0.85-1.38)$ & 0.517 \\
\hline AA & 15 & 2.98 & 33 & 2.22 & $\begin{array}{l}1.39(0.74- \\
2.58)\end{array}$ & 0.304 & $1.30(0.67-2.52)$ & 0.436 \\
\hline $\mathrm{GA}+\mathrm{AA}$ & 162 & 32.14 & 444 & 29.82 & $\begin{array}{c}1.12(0.90- \\
1.39)\end{array}$ & 0.327 & $1.10(0.87-1.39)$ & 0.417 \\
\hline $\mathrm{GG}+\mathrm{GA}$ & 489 & 97.02 & 1,456 & 97.78 & 1.00 & & 1.00 & \\
\hline AA & 15 & 2.98 & 33 & 2.22 & $\begin{array}{l}1.35(0.73- \\
2.51)\end{array}$ & 0.338 & $1.27(0.66-2.46)$ & 0.472 \\
\hline A allele & 177 & 17.56 & 477 & 16.02 & & & & \\
\hline \multicolumn{9}{|c|}{$L E P R \operatorname{rs} 1137101 \mathrm{G}>\mathrm{A}$} \\
\hline GG & 390 & 77.38 & 1,146 & 76.96 & 1.00 & & 1.00 & \\
\hline GA & 108 & 21.43 & 322 & 21.63 & $\begin{array}{l}0.98(0.77- \\
1.26)\end{array}$ & 0.898 & $0.91(0.70-1.18)$ & 0.473 \\
\hline AA & 6 & 1.19 & 21 & 1.41 & $\begin{array}{l}0.84(0.34- \\
2.09)\end{array}$ & 0.705 & $0.91(0.35-2.37)$ & 0.848 \\
\hline $\mathrm{GA}+\mathrm{AA}$ & 114 & 22.62 & 343 & 23.04 & $\begin{array}{l}0.98(0.77- \\
1.24)\end{array}$ & 0.848 & $0.91(0.70-1.18)$ & 0.468 \\
\hline $\mathrm{GG}+\mathrm{GA}$ & 498 & 98.81 & 1,468 & 98.59 & 1.00 & & 1.00 & \\
\hline AA & 6 & 1.19 & 21 & 1.41 & $\begin{array}{l}0.84(0.34- \\
2.10)\end{array}$ & 0.712 & $0.93(0.36-2.42)$ & 0.884 \\
\hline A allele & 120 & 11.90 & 364 & 12.22 & & & & \\
\hline \multicolumn{9}{|c|}{$L E P R \operatorname{rs} 6588147 \mathrm{G}>\mathrm{A}$} \\
\hline GG & 367 & 72.82 & 1,070 & 71.86 & 1.00 & & 1.00 & \\
\hline GA & 119 & 23.61 & 391 & 26.26 & $\begin{array}{l}0.89(0.70- \\
1.12)\end{array}$ & 0.316 & $0.85(0.66-1.09)$ & 0.199 \\
\hline AA & 18 & 3.57 & 28 & 1.88 & $\begin{array}{l}1.87(1.02- \\
3.43)\end{array}$ & 0.042 & $1.82(0.96-3.46)$ & 0.068 \\
\hline $\mathrm{GA}+\mathrm{AA}$ & 137 & 27.18 & 419 & 28.14 & $\begin{array}{l}0.95(0.76- \\
1.20)\end{array}$ & 0.680 & $0.91(0.72-1.16)$ & 0.465 \\
\hline $\mathrm{GG}+\mathrm{GA}$ & 486 & 96.43 & 1,461 & 98.12 & 1.00 & & 1.00 & \\
\hline AA & 18 & 3.57 & 28 & 1.88 & $\begin{array}{l}\text { 1.93(1.06- } \\
3.53)\end{array}$ & 0.031 & $1.90(1.00-3.61)$ & 0.049 \\
\hline A allele & 155 & 15.38 & 447 & 15.01 & & & & \\
\hline
\end{tabular}

a Adjusted for age, sex, BMI, alcohol use and smoking status.

Bold values are statistically significant $(P<0.05)$. 
Table 4: Stratified analyses between $L E P$ rs7799039 A>G polymorphism and ESCC risk by sex, age, BMI, smoking status and alcohol consumption

\begin{tabular}{|c|c|c|c|c|c|c|c|c|}
\hline \multirow{2}{*}{ Variable } & \multicolumn{3}{|c|}{$\begin{array}{c}L E P \text { rs7799039 A }>G \\
\quad \text { (case/control) }^{\mathrm{a}}\end{array}$} & \multicolumn{5}{|c|}{ Adjusted OR ${ }^{\mathrm{b}}(95 \% \mathrm{CI}) ; P$} \\
\hline & $\mathbf{A A}$ & $\mathbf{A G}$ & GG & $\mathbf{A A}$ & $\mathbf{A G}$ & GG & AG/GG & $\begin{array}{c}\text { GG vs. (AG/ } \\
\text { AA) }\end{array}$ \\
\hline \multicolumn{9}{|l|}{ Sex } \\
\hline Male & $222 / 581$ & $134 / 425$ & $19 / 72$ & 1.00 & $\begin{array}{c}0.81(0.62-1.06) \\
P: 0.117\end{array}$ & $\begin{array}{c}0.60(0.33- \\
1.06) \\
P: 0.079\end{array}$ & $\begin{array}{c}0.77(0.60- \\
1.00) \\
P: 0.052\end{array}$ & $\begin{array}{c}0.65(0.37- \\
1.15) \\
P: 0.136\end{array}$ \\
\hline Female & $69 / 216$ & $50 / 166$ & $10 / 29$ & 1.00 & $\begin{array}{c}0.97(0.63-1.50) \\
P: 0.901\end{array}$ & $\begin{array}{c}1.324(0.60- \\
2.97) ; \\
P: 0.475\end{array}$ & $\begin{array}{c}1.03(0.68- \\
1.55) \\
P: 0.897\end{array}$ & $\begin{array}{c}1.36(0.62- \\
2.95) \\
P: 0.442\end{array}$ \\
\hline \multicolumn{9}{|l|}{ Age } \\
\hline$<63$ & $139 / 395$ & 79/306 & $18 / 46$ & 1.00 & $\begin{array}{c}0.78(0.56-1.11) \\
P: 0.166\end{array}$ & $\begin{array}{c}1.31(0.69- \\
2.50) \\
P: 0.409\end{array}$ & $\begin{array}{c}0.84(0.60- \\
1.16) \\
P: 0.282\end{array}$ & $\begin{array}{c}1.43(0.76- \\
2.69) \\
P: 0.263\end{array}$ \\
\hline$\geq 63$ & $152 / 402$ & $105 / 285$ & $11 / 55$ & 1.00 & $\begin{array}{c}0.95(0.70-1.29) \\
P: 0.737\end{array}$ & $\begin{array}{c}0.47(0.23- \\
0.95) \\
P: 0.035\end{array}$ & $\begin{array}{c}0.88(0.65- \\
1.18) \\
P: 0.395\end{array}$ & $\begin{array}{c}0.48(0.24- \\
0.96) ; \\
P: 0.038\end{array}$ \\
\hline \multicolumn{9}{|l|}{$\begin{array}{l}\text { Smoking } \\
\text { status }\end{array}$} \\
\hline Never & $146 / 589$ & $83 / 427$ & $16 / 70$ & 1.00 & $\begin{array}{c}0.79(0.59-1.08) \\
P: 0.135\end{array}$ & $\begin{array}{c}0.99(0.55- \\
1.78) \\
P: 0.970\end{array}$ & $\begin{array}{c}0.83(0.62- \\
1.10) \\
P: 0.190\end{array}$ & $\begin{array}{c}1.09(0.61- \\
1.93) \\
P: 0.779\end{array}$ \\
\hline Ever & $145 / 208$ & $101 / 164$ & $13 / 31$ & 1.00 & $\begin{array}{c}0.92(0.65-1.31) \\
P: 0.637\end{array}$ & $\begin{array}{c}0.49(0.23- \\
1.02) \\
P: 0.057\end{array}$ & $\begin{array}{c}0.84(0.60- \\
1.18) \\
P: 0.306\end{array}$ & $\begin{array}{c}0.50(0.24- \\
1.04) \\
P: 0.063\end{array}$ \\
\hline \multicolumn{9}{|c|}{ Alcohol consumption } \\
\hline Never & $198 / 706$ & $122 / 526$ & $18 / 91$ & 1.00 & $\begin{array}{c}0.82(0.63-1.06) \\
P: 0.135\end{array}$ & $\begin{array}{c}0.72(0.42- \\
1.23) \\
P: 0.229\end{array}$ & $\begin{array}{c}0.81(0.63- \\
1.04) \\
P: 0.097\end{array}$ & $\begin{array}{c}0.78(0.46- \\
1.33) \\
P: 0.359\end{array}$ \\
\hline Ever & 93/91 & $62 / 65$ & $11 / 10$ & 1.00 & $\begin{array}{c}1.06(0.64-1.77) \\
P: 0.820\end{array}$ & $\begin{array}{c}0.75(0.28- \\
1.96) ; \\
P: 0.552\end{array}$ & $\begin{array}{c}0.99(0.61- \\
1.60) \\
P: 0.955\end{array}$ & $\begin{array}{c}0.72(0.28- \\
1.85) \\
P: 0.492\end{array}$ \\
\hline \multicolumn{9}{|c|}{ BMI $\left(\mathrm{kg} / \mathrm{m}^{2}\right)$} \\
\hline$<24$ & $210 / 436$ & $137 / 285$ & $20 / 53$ & 1.00 & $\begin{array}{c}0.96(0.72-1.26) \\
P: 0.744\end{array}$ & $\begin{array}{c}0.63(0.35- \\
1.13) \\
P: 0.118\end{array}$ & $\begin{array}{c}0.90(0.69- \\
1.18) \\
P: 0.458\end{array}$ & $\begin{array}{c}0.64(0.36- \\
1.13) \\
P: 0.126\end{array}$ \\
\hline$\geq 24$ & $81 / 361$ & $47 / 306$ & $9 / 48$ & 1.00 & $\begin{array}{c}0.66(0.45-0.99) \\
P: 0.044\end{array}$ & $\begin{array}{c}0.93(0.43- \\
1.99) \\
P: 0.847\end{array}$ & $\begin{array}{c}0.69(0.48- \\
1.01) \\
P: 0.058\end{array}$ & $\begin{array}{c}1.09(0.52- \\
2.31) \\
P: 0.816\end{array}$ \\
\hline
\end{tabular}

${ }^{a}$ For $L E P$ rs7799039 A>G, the genotyping was successful in 507 (99.41\%) ESCC cases, and 1,496 (99.53\%) controls. ${ }^{\mathrm{b}}$ Adjusted for multiple comparisons [age, sex, BMI, smoking status and alcohol consumption (besides stratified factors accordingly)] in a logistic regression model. 
Table 5: Stratified analyses between $L E P R$ rs1137101 G>A polymorphism and ESCC risk by sex, age, BMI, smoking status and alcohol consumption

\begin{tabular}{|c|c|c|c|c|c|c|c|c|}
\hline \multirow{2}{*}{ Variable } & \multicolumn{3}{|c|}{$\begin{array}{c}L E P R \text { rs1137101 G>A } \\
\left(_{(\text {case/control) }}{ }^{a}\right.\end{array}$} & \multicolumn{5}{|c|}{ Adjusted OR ${ }^{\mathrm{b}}(95 \%$ CI $) ; P$} \\
\hline & GG & GA & $\mathbf{A A}$ & GG & GA & $\mathbf{A A}$ & GA/AA & $\begin{array}{l}\text { AA vs. (GA/ } \\
\text { GG) }\end{array}$ \\
\hline \multicolumn{9}{|l|}{ Sex } \\
\hline Male & $292 / 832$ & $78 / 235$ & $5 / 11$ & 1.00 & $\begin{array}{c}0.84(0.61- \\
1.15) ; \\
P: 0.275\end{array}$ & $\begin{array}{c}1.52(0.49- \\
4.75) \\
P: 0.473\end{array}$ & $\begin{array}{c}0.87(0.64- \\
1.18) \\
P: 0.353\end{array}$ & $\begin{array}{c}1.57(0.50-4.91) \\
P: 0.435\end{array}$ \\
\hline Female & $98 / 314$ & $30 / 87$ & $1 / 10$ & 1.00 & $\begin{array}{c}1.11(0.68- \\
1.81) ; \\
P: 0.686\end{array}$ & $\begin{array}{c}0.27(0.03- \\
2.24) \\
P: 0.226\end{array}$ & $\begin{array}{c}1.02(0.63- \\
1.65) \\
P: 0.943\end{array}$ & $\begin{array}{c}0.27(0.03-2.20) \\
P: 0.220\end{array}$ \\
\hline \multicolumn{9}{|l|}{ Age } \\
\hline$<63$ & $177 / 579$ & $55 / 157$ & $4 / 11$ & 1.00 & $\begin{array}{c}1.06(0.72- \\
1.57) ; \\
P: 0.772\end{array}$ & $\begin{array}{c}1.77(0.51- \\
6.13) ; \\
P: 0.370\end{array}$ & $\begin{array}{c}1.09(0.74- \\
1.59) \\
P: 0.666\end{array}$ & $\begin{array}{c}1.73(0.50-5.98) ; \\
P: 0.387\end{array}$ \\
\hline$\geq 63$ & $213 / 567$ & $53 / 165$ & $2 / 10$ & 1.00 & $\begin{array}{c}0.75(0.52- \\
1.08) \\
P: 0.123\end{array}$ & $\begin{array}{c}0.43(0.09- \\
2.02) \\
P: 0.283\end{array}$ & $\begin{array}{c}0.74(0.52- \\
1.06) \\
P: 0.097\end{array}$ & $\begin{array}{c}0.46(0.10-2.16) \\
P: 0.323\end{array}$ \\
\hline \multicolumn{9}{|c|}{ Smoking status } \\
\hline Never & $186 / 848$ & $56 / 221$ & $3 / 17$ & 1.00 & $\begin{array}{c}1.15(0.82- \\
1.61) \\
P: 0.432\end{array}$ & $\begin{array}{c}0.72(0.20- \\
2.56) \\
P: 0.613\end{array}$ & $\begin{array}{c}1.12(0.80- \\
1.57) \\
P: 0.504\end{array}$ & $\begin{array}{c}0.70(0.20-2.49) \\
P: 0.585\end{array}$ \\
\hline Ever & $204 / 298$ & $52 / 101$ & $3 / 4$ & 1.00 & $\begin{array}{c}0.66(0.44- \\
1.00) ; \\
P: 0.049\end{array}$ & $\begin{array}{c}1.51(0.30- \\
7.58) \\
P: 0.616\end{array}$ & $\begin{array}{c}0.68(0.46- \\
1.02) \\
P: 0.063\end{array}$ & $\begin{array}{c}1.65(0.33-8.24) \text {; } \\
P: 0.543\end{array}$ \\
\hline \multicolumn{9}{|c|}{ Alcohol consumption } \\
\hline Never & $260 / 1,028$ & $73 / 276$ & $5 / 19$ & 1.00 & $\begin{array}{c}1.00(0.74- \\
1.35) \\
P: 0.999\end{array}$ & $\begin{array}{c}1.04(0.37- \\
2.89) \\
P: 0.943\end{array}$ & $\begin{array}{c}1.01(0.75- \\
1.35) \\
P: 0.953\end{array}$ & $\begin{array}{c}1.04(0.38-2.89) ; \\
P: 0.939\end{array}$ \\
\hline Ever & $130 / 118$ & $35 / 46$ & $1 / 2$ & 1.00 & $\begin{array}{c}0.54(0.31- \\
0.95) ; \\
P: 0.031\end{array}$ & $\begin{array}{c}0.56(0.04- \\
8.70) \\
P: 0.679\end{array}$ & $\begin{array}{c}0.54(0.31- \\
0.93) ; \\
P: 0.027\end{array}$ & $\begin{array}{c}0.64(0.04-9.68) \\
P: 0.750\end{array}$ \\
\hline \multicolumn{9}{|c|}{ BMI $\left(\mathrm{kg} / \mathrm{m}^{2}\right)$} \\
\hline$<24$ & $279 / 600$ & $83 / 165$ & $5 / 9$ & 1.00 & $\begin{array}{c}0.99(0.72- \\
1.36) \\
P: 0.930\end{array}$ & $\begin{array}{c}1.32(0.42- \\
4.18) \\
P: 0.633\end{array}$ & $\begin{array}{c}1.01(0.74- \\
1.38) \\
P: 0.972\end{array}$ & $\begin{array}{c}1.33(0.42-4.20) ; \\
P: 0.623\end{array}$ \\
\hline$\geq 24$ & $111 / 546$ & $25 / 157$ & $1 / 12$ & 1.00 & $\begin{array}{c}0.76(0.47- \\
1.22) ; \\
P: 0.250\end{array}$ & $\begin{array}{c}0.39(0.05- \\
3.12) \\
P: 0.376\end{array}$ & $\begin{array}{c}0.73(0.45- \\
1.16) \\
P: 0.183\end{array}$ & $\begin{array}{c}0.41(0.05-3.29) \\
P: 0.405\end{array}$ \\
\hline
\end{tabular}

${ }^{a}$ For $L E P R$ rs $1137101 \mathrm{G}>\mathrm{A}$, the genotyping was successful in 507 (99.41\%) ESCC cases, and 1,496 (99.53\%) controls. ${ }^{\mathrm{b}}$ Adjusted for multiple comparisons [age, sex, BMI, smoking status and alcohol consumption (besides stratified factors accordingly)] in a logistic regression model. 
Table 6: Stratified analyses between $L E P R$ rs6588147 G>A polymorphism and ESCC risk by sex, age, BMI, smoking status and alcohol consumption

\begin{tabular}{|c|c|c|c|c|c|c|c|c|}
\hline \multirow{2}{*}{ Variable } & \multicolumn{3}{|c|}{$\begin{array}{c}L E P R \text { rs6588147 G }>A \\
\left(^{(\text {case } / \text { control) }}\right)^{\mathrm{a}}\end{array}$} & \multicolumn{5}{|c|}{ Adjusted OR $^{\mathrm{b}}(95 \% \mathrm{CI}) ; P$} \\
\hline & GG & GA & $\mathbf{A A}$ & GG & GA & $\mathbf{A A}$ & GA/AA & $\begin{array}{c}\text { AA vs. } \\
\text { (GA/GG) }\end{array}$ \\
\hline \multicolumn{9}{|l|}{ Sex } \\
\hline Male & $267 / 769$ & $94 / 290$ & $14 / 19$ & 1.00 & $\begin{array}{c}0.89(0.67-1.20) \\
P: 0.449\end{array}$ & $\begin{array}{c}2.19(1.02-4.67) \\
P: 0.044\end{array}$ & $\begin{array}{c}0.97(0.73-1.29) \\
P: 0.834\end{array}$ & $\begin{array}{c}2.26(1.06- \\
4.80) \\
P: 0.035\end{array}$ \\
\hline Female & $100 / 301$ & $25 / 101$ & $4 / 9$ & 1.00 & $\begin{array}{c}0.72(0.43-1.20) \\
P: 0.204\end{array}$ & $\begin{array}{c}1.19(0.34-4.22) \\
P: 0.785\end{array}$ & $\begin{array}{c}0.76(0.47-1.24) \\
P: 0.274\end{array}$ & $\begin{array}{c}1.29(0.37- \\
4.55) ; \\
P: 0.688\end{array}$ \\
\hline \multicolumn{9}{|l|}{ Age } \\
\hline$<63$ & $168 / 527$ & $59 / 206$ & $9 / 14$ & 1.00 & $\begin{array}{c}0.80(0.55-1.16) \\
P: 0.233\end{array}$ & $\begin{array}{c}2.58(1.00-6.62) \\
P: 0.049\end{array}$ & $\begin{array}{c}0.88(0.62-1.26) \\
P: 0.484\end{array}$ & $\begin{array}{c}2.71(1.06- \\
6.91) ; \\
P: 0.038\end{array}$ \\
\hline$\geq 63$ & $199 / 543$ & $60 / 185$ & $9 / 14$ & 1.00 & $\begin{array}{c}0.84(0.59-1.20) \\
P: 0.339\end{array}$ & $\begin{array}{c}1.40(0.58-3.39) \\
P: 0.458\end{array}$ & $\begin{array}{c}0.90(0.64-1.26) \\
P: 0.534\end{array}$ & $\begin{array}{c}1.48(0.61- \\
3.56) ; \\
P: 0.386\end{array}$ \\
\hline \multicolumn{9}{|c|}{ Smoking status } \\
\hline Never & $180 / 787$ & $56 / 279$ & $9 / 20$ & 1.00 & $\begin{array}{c}0.89(0.63-1.24) \\
P: 0.486\end{array}$ & $\begin{array}{c}1.88(0.82-4.31) \\
P: 0.139\end{array}$ & $\begin{array}{c}0.96(0.70-1.32) \\
P: 0.807\end{array}$ & $\begin{array}{c}1.94(0.85- \\
4.44) ; \\
P: 0.117\end{array}$ \\
\hline Ever & $187 / 283$ & $63 / 112$ & $9 / 8$ & 1.00 & $\begin{array}{c}0.80(0.54-1.17) \\
P: 0.248\end{array}$ & $\begin{array}{c}2.00(0.71-5.66) \\
P: 0.191\end{array}$ & $\begin{array}{c}0.86(0.59-1.25) \\
P: 0.438\end{array}$ & $\begin{array}{c}2.12(0.75- \\
5.97) ; \\
P: 0.155\end{array}$ \\
\hline \multicolumn{9}{|c|}{$\begin{array}{l}\text { Alcohol } \\
\text { consumption }\end{array}$} \\
\hline Never & $245 / 961$ & $80 / 335$ & $13 / 27$ & 1.00 & $\begin{array}{c}0.92(0.69-1.23) \\
P: 0.590\end{array}$ & $\begin{array}{c}1.69(0.84-3.40) \\
P: 0.145\end{array}$ & $\begin{array}{c}0.99(0.75-1.30) \\
P: 0.944\end{array}$ & $\begin{array}{c}1.73(0.86- \\
3.47) ; \\
P: 0.124\end{array}$ \\
\hline Ever & $122 / 109$ & $39 / 56$ & $5 / 1$ & 1.00 & $\begin{array}{c}0.54(0.31-0.92) \\
P: 0.024\end{array}$ & $\begin{array}{c}5.03(0.48-52.46) \\
P: 0.177\end{array}$ & $\begin{array}{c}0.60(0.35-1.01) \\
P: 0.056\end{array}$ & $\begin{array}{c}5.79(0.56- \\
59.52) ; \\
P: 0.139\end{array}$ \\
\hline \multicolumn{9}{|c|}{ BMI (kg/m²) } \\
\hline$<24$ & $261 / 552$ & $92 / 204$ & $14 / 18$ & 1.00 & $\begin{array}{c}0.94(0.69-1.28) \\
P: 0.700\end{array}$ & $\begin{array}{c}1.79(0.84-3.82) \\
P: 0.130\end{array}$ & $\begin{array}{c}1.01(0.76-1.36) \\
P: 0.936\end{array}$ & $\begin{array}{c}1.83(0.86- \\
3.89) ; \\
P: 0.115\end{array}$ \\
\hline$\geq 24$ & $106 / 518$ & $27 / 187$ & $4 / 10$ & 1.00 & $\begin{array}{c}0.67(0.42-1.07) \\
P: 0.093\end{array}$ & $\begin{array}{c}1.96(0.59-6.59) \\
P: 0.275\end{array}$ & $\begin{array}{c}0.73(0.47-1.14) \\
P: 0.168\end{array}$ & $\begin{array}{c}2.14(0.64- \\
7.17) ; \\
P: 0.215\end{array}$ \\
\hline
\end{tabular}

${ }^{a}$ For $L E P R$ rs $1137101 \mathrm{G}>\mathrm{A}$, the genotyping was successful in 507 (99.41\%) ESCC cases, and 1,496 (99.53\%) controls. ${ }^{\mathrm{b}}$ Adjusted for multiple comparisons [age, sex, BMI, smoking status and alcohol consumption (besides stratified factors accordingly)] in a logistic regression model. 
In addition, after a logistic regression analysis, we found that TCF7L2 rs7903146C $>$ T, rs290481 $\mathrm{T}>\mathrm{C}, L E P$ rs2167270 $\mathrm{G}>\mathrm{A}$ and $L E P R$ rs1137100 $\mathrm{G}>\mathrm{A}$ polymorphisms were not associated with the risk of ESCC in any subgroup (data not shown).

\section{DISCUSSION}

The pathogenesis of ESCC was very complex. Multiple factors (e.g. a number of genetic and environmental factors) may contribute to the etiology of ESCC. Understanding of the individual's heredity background may be helpful for the prevention and treatment of ESCC. In this study, we selected energy metabolism and insulin-sensibility relative gene (TCF7L2, $L E P$ and $L E P R$ ) polymorphisms and focused on their susceptibility to ESCC. The association between $L E P R$ rs $6588147 \mathrm{G}>\mathrm{A}$ polymorphism and the increased risk of overall ESCC was identified. We also found that $L E P R$ rs $6588147 \mathrm{G}>\mathrm{A}$ polymorphism increased the risk of ESCC in $<63$ years and male subgroups. LEP rs7799039 A $>\mathrm{G}$ was associated with the risk of ESCC in $\geq 63$ years and $\mathrm{BMI} \geq 24 \mathrm{~kg} / \mathrm{m}^{2}$ subgroups. In addition, LEPR $\mathrm{rs} 1137101$ $\mathrm{G}>\mathrm{A}$ polymorphism decreased the risk of ESCC in ever smoking and ever drinking subgroups.

There was a difference in the LEPR rs6588147 $\mathrm{G}>\mathrm{A}$ polymorphism between overall ESCC patients and non-cancer controls. The LEPR rs6588147 AA genotype were higher in ESCC patients compared with controls, indicating that $L E P R$ rs6588147 AA genotype may contribute to esophageal carcinogenesis. The $L E P R$ rs6588147 $\mathrm{G}>\mathrm{A}$ polymorphism is located on intron of $L E P R$ gene. It may be difficult to interpret the exact function of intronic polymorphism. However, the possible interpretations may be as follows. The intronic polymorphism rs6588147 $\mathrm{G}>\mathrm{A}$ is located near the regulatory components or splice acceptor site, where any slight variant may lead to the disruption of the splice site and induce aberrant splicing [29]. This SNP probably influences the expression of the LEPR protein by altering mRNA splicing. However, we found that $L E P R$ rs6588147 AA genotype may decrease the risk of ESCC in ever drinking subgroup. These findings seemed to be controversial. The probable reason might be due to the limited sample size in this subgroup, which could generate an unauthentic results.

LEP is mainly secreted by adipose tissue, and has been suggested to promote tumor growth [30]. Some studies indicated that the serum LEP level was significantly higher in breast cancer patients compared with which in controls both pre-menopausal and postmenopausal [31, 32]. A number of studies have found that LEP may play vital roles in cell proliferation, apoptosis, cell migration and angiogenesis [33, 34]. Results of several meta-analyses suggested that LEP rs $7799039 \mathrm{G}$ allele might decrease the risk of multiple cancers $[24,25$,
35-37]. However, there was only one study focused on the relationship between $L E P$ rs7799039 A > G polymorphism and cancer risk in Asian populations. Thus, the association of this polymorphism with cancer risk might be unclear in Asians. In this study, we conducted a case-control study focused on the association between LEP rs7799039 $\mathrm{A}>\mathrm{G}$ polymorphism and ESCC risk with a relatively large sample size. We found $L E P$ rs7799039 A $>$ G was associated with the decreased risk of ESCC in $\geq 63$ years and $\mathrm{BMI} \geq 24 \mathrm{~kg} / \mathrm{m}^{2}$ subgroups. These findings were very similar to the results of previous studies. Hoffsted et al. reported that individuals carried the LEP rs7799039 AA genotype had higher serum LEP levels than those who carried the LEP rs7799039AG or GG genotypes [27]. In this study, we found that $L E P$ rs $7799039 A>G$ polymorphism was a protective factor for ESCC, suggesting the presence of the LEP rs7799039 G allele, which is associated with the decreased level of LEP, might decrease the risk of ESCC.

Several case-control study focused on the relationship of $L E P R$ rs $1137101 \mathrm{G}>\mathrm{A}$ polymorphism and the risk of cancer. Recently, results of two meta-analyses indicated that this SNP was not associated with the risk of overall cancer $[37,38]$. In addition, most of these studies conducted on Caucasian population. The evidence of the association between $L E P R$ rs $1137101 \mathrm{G}>$ A polymorphism and cancer risk was insufficient in Asians. A previous study suggested that LEPR rs1137101 G>A polymorphism might be associated with variation in binding with LEP and, as such, inter-individual differences in serum LEP levels [39]. Just as we mentioned above, LEP may affect cell proliferation, apoptosis, cell migration and angiogenesis. LEPR rs $1137101 \mathrm{G}>\mathrm{A}$ polymorphism may alter the susceptibility of cancer by influencing the ability of binding with LEP. Thus, we aimed to examine the potential association of this polymorphism with the risk of ESCC in Eastern Chinese Han subjects. We found that the $L E P R$ rs1137101 G>A polymorphism decreased ESCC risk in ever drinking and ever smoking subgroups. In the future, function of LEPR rs $1137101 \mathrm{G}>\mathrm{A}$ polymorphism should be further explored to confirm these primary findings in ESCC.

Our study had several limitations. Firstly, ESCC patients and controls were enrolled from two hospitals of Jiangsu University and Fujian Medical University and might therefore not be full-representative of the general Eastern Chinese Han population; the possible bias might lead to spurious findings. Secondly, for the limited ESCC patients recruited in this study, this study might have insufficient power to observe the potential relationships. Thirdly, because we only selected some functional polymorphisms in TCF7L2, $L E P$ and $L E P R$ gene, a finemapping case-control studies should be conducted in the future. Finally, for lack of some important risk factors, the interactive effect between gene-gene and geneenvironment was not further analyzed. 
In summary, our findings suggest that LEPR rs6588147 $\mathrm{G}>\mathrm{A}$ polymorphism is associated with the increased risk of ESCC in Eastern Chinese Han population. However, the results of this case-control study highlight that $L E P$ rs7799039 $\mathrm{A}>\mathrm{G}$ and $L E P R$ rs 1137101 $\mathrm{G}>\mathrm{A}$ polymorphisms may decrease the risk of ESCC. A fine-mapping study with large sample size and functional exploration is needed to confirm our findings.

\section{MATERIALS AND METHODS}

\section{Subjects}

A total number of 507 ESCC patients and 1,496 non-cancer controls were enrolled in this study. The ESCC patients were from the Affiliated People's Hospital, Jiangsu University and the Affiliated Union Hospital, Fujian Medical University between August 2013 and December 2016. The diagnosis of ESCC was confirmed based on pathological examination. At the same time, the controls were recruited from physical examination center in these hospitals with sex and age matched. Each subject signed an informed written consent. This study was approved by the Institutional Review Board of Jiangsu University and Fujian Medical University for human subjects (No. SQ20140030, K201408, respectively). When each subject was interviewed, a questionnaire was used to obtain demographic variables and risk factors. And weight and height were also measured. In this study, a BMI $\geq 24$ was considered as the criteria for obesity and overweight $[40,41]$.

\section{DNA extraction and genotyping}

Genomic DNA was carefully isolated from EDTAanticoagulated blood of recipients by using a Promega DNA blood mini kit (Promega, Madison, USA). TCF7L2 rs7903146C $>$ T, rs290481 $\mathrm{T}>\mathrm{C}, L E P$ rs7799039 A $>\mathrm{G}$, rs2167270 $\mathrm{G}>\mathrm{A}$ and $L E P R$ rs1137100 $\mathrm{G}>\mathrm{A}$, rs1137101 $\mathrm{G}>\mathrm{A}$ and rs6588147 $\mathrm{G}>\mathrm{A}$ genotypes were assessed by the SNPscan ${ }^{\mathrm{TM}}$ kit (Gnensky Biotechologies Inc., Shanghai, China), which is a double ligation and multiplex fluorescence PCR [42]. For quality control, eighty DNA samples $(4 \%)$ were randomly selected and genotyped by different colleague. The genotypes of TCF $7 L 2, L E P$ and $L E P R$ polymorphisms were confirmed.

\section{Statistical analysis}

Continuous variables (e.g. age, height, weight and BMI) are expressed as mean \pm standard deviation (SD). Comparisons between ESCC patients and controls were carried out with Student's t-test. The categorical variables (e.g. TCF7L2, LEP and LEPR genotypes, sex, age, BMI, smoking and drinking status) were compared with Chisquare test $\left(\chi^{2}\right)$. Deviations from the HWE for TCF7L2, $L E P$ and $L E P R$ genotypes distribution in controls were evaluated by an internet-based calculator (http://ihg.gsf. de/cgi-bin/hw/hwa1.pl) [43-49]. The relationships of TCF7L2 rs7903146C > T, rs290481 T>C, LEP rs7799039 $\mathrm{A}>\mathrm{G}, \quad$ rs2167270 $\mathrm{G}>\mathrm{A}$ and $L E P R \quad$ rs1137100 $\mathrm{G}>\mathrm{A}$, rs1137101 $\mathrm{G}>\mathrm{A}$ and $\mathrm{rs} 6588147 \mathrm{G}>\mathrm{A}$ polymorphisms with ESCC susceptibility were evaluated by crude ORs and 95\% CIs. Multivariate linear regression adjusted for age, sex, BMI, alcohol use and smoking status was used to determine the relationships between TCF7L2 rs7903146C $>$ T, rs290481 $\mathrm{T}>\mathrm{C}, L E P$ rs7799039 A $>\mathrm{G}$, rs2167270 $\mathrm{G}>\mathrm{A}$ and $L E P R$ rs1137100 $\mathrm{G}>\mathrm{A}$, rs 1137101 $\mathrm{G}>\mathrm{A}$ and rs6588147 $\mathrm{G}>\mathrm{A}$ polymorphisms and ESCC risk with quantitative traits. Data analysis was conducted with SAS software for windows (Version 9.4, SAS Institute, Cary, NC). A $P<0.05$ (two-tailed) was accepted as the criterion of statistical significance.

\section{ACKNOWLEDGMENTS}

We appreciate all subjects who participated in this study. We wish to thank Dr. Yan Liu (Genesky Biotechnologies Inc., Shanghai, China) for technical support.

\section{CONFLICTS OF INTEREST}

The authors have no potential financial conflicts of interest.

\section{GRANT SUPPORT}

This study was supported in part by Natural Science Foundation of Universities and Colleges of Jiangsu Province (Grant No. 16KJB310002), Senior Talents Scientific Research Foundation of Jiangsu University (Grant No. 16JDG066), Young and Middle-aged Talent Training Project of Health Development Planning Commission in Fujian Province (2016-ZQN-25 and 2014-ZQN-JC-11), Medical Innovation Project of Fujian Province (2014-CX-15 and 2014-CX-18), Nursery Garden Project of Fujian Medical University (2015MP020) and Science and Technology Project of Fujian Province (2060203).

\section{REFERENCES}

1. Chen W, Zheng R, Baade PD, Zhang S, Zeng H, Bray F, Jemal A, Yu XQ, He J. Cancer statistics in China, 2015. CA Cancer J Clin. 2016; 66:115-132.

2. Pischon T, Nimptsch K. Obesity and risk of cancer: an introductory overview. Recent Results Cancer Res. 2016; 208:1-15.

3. Peng F, Hu D, Lin X, Chen G, Liang B, Zhang H, Dong $\mathrm{X}$, Lin J, Zheng X, Niu W. Analysis of preoperative metabolic risk factors affecting the prognosis of patients 
with esophageal squamous cell carcinoma: the Fujian prospective investigation of cancer (FIESTA) study. EBioMedicine. 2017; 16:115-123.

4. Duval A, Busson-Leconiat M, Berger R, Hamelin R. Assignment of the TCF-4 gene (TCF7L2) to human chromosome band 10q25.3. Cytogenet Cell Genet. 2000; 88:264-265.

5. Damcott CM, Pollin TI, Reinhart LJ, Ott SH, Shen H, Silver KD, Mitchell BD, Shuldiner AR. Polymorphisms in the transcription factor 7-like 2 (TCF7L2) gene are associated with type 2 diabetes in the Amish: replication and evidence for a role in both insulin secretion and insulin resistance. Diabetes. 2006; 55:2654-2659.

6. Cavallo RA, Cox RT, Moline MM, Roose J, Polevoy GA, Clevers H, Peifer M, Bejsovec A. Drosophila Tcf and Groucho interact to repress Wingless signalling activity. Nature. 1998; 395:604-608.

7. MacDonald BT, Tamai $\mathrm{K}$, He X. Wnt/beta-catenin signaling: components, mechanisms, and diseases. Dev Cell. 2009; 17:9-26.

8. Ishiguro H, Wakasugi T, Terashita Y, Sakamoto N, Tanaka T, Sagawa H, Okubo T, Takeyama H. Nuclear expression of TCF4/TCF7L2 is correlated with poor prognosis in patients with esophageal squamous cell carcinoma. Cell Mol Biol Lett. 2016; 21:5.

9. Zhou Y, Oskolkov N, Shcherbina L, Ratti J, Kock KH, Su J, Martin B, Oskolkova MZ, Goransson O, Bacon J, Li W, Bucciarelli S, Cilio C, et al. HMGB1 binds to the rs 7903146 locus in TCF7L2 in human pancreatic islets. Mol Cell Endocrinol. 2016; 430:138-145.

10. Connor AE, Baumgartner RN, Baumgartner KB, Kerber RA, Pinkston C, John EM, Torres-Mejia G, Hines L, Giuliano A, Wolff RK, Slattery ML. Associations between TCF7L2 polymorphisms and risk of breast cancer among Hispanic and non-Hispanic white women: the Breast Cancer Health Disparities Study. Breast Cancer Res Treat. 2012; 136:593-602.

11. Wang F, Jiang L, Li J, Yu X, Li M, Wu G, Yu Z, Zhou $\mathrm{K}$, Chu H, Zhai H. Association between TCF7L2 polymorphisms and breast cancer susceptibility: a metaanalysis. Int J Clin Exp Med. 2015; 8:9355-9361.

12. Ling Q, Dong F, Geng L, Liu Z, Xie H, Xu X, Zheng S. Impacts of TCF7L2 gene polymorphisms on the susceptibility of hepatogenous diabetes and hepatocellular carcinoma in cirrhotic patients. Gene. 2013; 522:214-218.

13. Unger RH, Zhou YT, Orci L. Regulation of fatty acid homeostasis in cells: novel role of leptin. Proc Natl Acad Sci U S A. 1999; 96:2327-2332.

14. Yiannakouris N, Yannakoulia M, Melistas L, Chan JL, Klimis-Zacas D, Mantzoros CS. The Q223R polymorphism of the leptin receptor gene is significantly associated with obesity and predicts a small percentage of body weight and body composition variability. J Clin Endocrinol Metab. $2001 ; 86: 4434-4439$.
15. Han HR, Ryu HJ, Cha HS, Go MJ, Ahn Y, Koo BK, Cho YM, Lee HK, Cho NH, Shin C, Shin HD, Kimm K, $\mathrm{Kim} \mathrm{HL}$, et al. Genetic variations in the leptin and leptin receptor genes are associated with type 2 diabetes mellitus and metabolic traits in the Korean female population. Clin Genet. 2008; 74:105-115.

16. Marcello MA, Calixto AR, de Almeida JF, Martins MB, Cunha LL, Cavalari CA, Etchebehere EC, da Assumpcao LV, Geloneze B, Carvalho AL, Ward LS. Polymorphism in LEP and LEPR may modify leptin levels and represent risk factors for thyroid cancer. Int J Endocrinol. 2015; 2015:173218.

17. Hussain SR, Naqvi H, Gupta S, Mahdi AA, Kumari P, Waseem M, Ahmad MK. A study on oncogenic role of leptin and leptin receptor in oral squamous cell. Tumour Biol. 2015; 36:6515-6523.

18. Mantzoros CS, Moschos SJ. Leptin: in search of role(s) in human physiology and pathophysiology. Clin Endocrinol (Oxf). 1998; 49:551-567.

19. Alshaker H, Krell J, Frampton AE, Waxman J, Blyuss O, Zaikin A, Winkler M, Stebbing J, Yague E, Pchejetski D. Leptin induces upregulation of sphingosine kinase 1 in oestrogen receptor-negative breast cancer via Src family kinase-mediated, janus kinase 2-independent pathway. Breast Cancer Res. 2014; 16:426.

20. Niu J, Jiang L, Guo W, Shao L, Liu Y, Wang L. The association between leptin level and breast cancer: a metaanalysis. PLoS One. 2013; 8:e67349.

21. Kato S, Abarzua-Catalan L, Trigo C, Delpiano A, Sanhueza C, Garcia K, Ibanez C, Hormazabal K, Diaz D, Branes J, Castellon E, Bravo E, Owen G, Cuello MA. Leptin stimulates migration and invasion and maintains cancer stem-like properties in ovarian cancer cells: an explanation for poor outcomes in obese women. Oncotarget. 2015; 6:21100-21119. https://doi.org/10.18632/oncotarget.4228.

22. Oba J, Wei W, Gershenwald JE, Johnson MM, Wyatt CM, Ellerhorst JA, Grimm EA. Elevated serum leptin levels are associated with an increased risk of sentinel lymph node metastasis in cutaneous melanoma. Medicine. 2016; 95:e3073.

23. Liu P, Shi H, Huang C, Shu H, Liu R, Yang Y, Gong J, Yang Y, Cai M. Association of LEP A19G polymorphism with cancer risk: a systematic review and pooled analysis. Tumour Biol. 2014; 35:8133-8141.

24. Yang Y, Liu P, Guo F, Liu R, Yang Y, Huang C, Shu H, Gong J, Cai M. Genetic G2548A polymorphism of leptin gene and risk of cancer: a meta-analysis of 6860 cases and 7956 controls. J BUON. 2014; 19:1096-1104.

25. Liu Y, Wu H, Zhu Y, Gao Y. Genetic association between leptin-2548G/A polymorphism and risk of cancer: a meta analysis. Int J Clin Exp Med. 2015; 8:448-455.

26. Doecke JD, Zhao ZZ, Stark MS, Green AC, Hayward NK, Montgomery GW, Webb PM, Whiteman DC; Australian Cancer Study. Single nucleotide polymorphisms in 
obesity-related genes and the risk of esophageal cancers. Cancer Epidemiol Biomarkers Prev. 2008; 17:1007-1012.

27. Hoffstedt J, Eriksson P, Mottagui-Tabar S, Arner P. A polymorphism in the leptin promoter region (-2548 G/A) influences gene expression and adipose tissue secretion of leptin. Horm Metab Res. 2002; 34:355-359.

28. Slattery ML, Wolff RK, Herrick J, Caan BJ, Potter JD. Leptin and leptin receptor genotypes and colon cancer: gene-gene and gene-lifestyle interactions. Int J Cancer. 2008; 122:1611-1617.

29. Gazave E, Marques-Bonet T, Fernando O, Charlesworth B, Navarro A. Patterns and rates of intron divergence between humans and chimpanzees. Genome Biol. 2007; 8:R21.

30. Housa D, Housova J, Vernerova Z, Haluzik M. Adipocytokines and cancer. Physiol Res. 2006; 55:233-244.

31. Mohammadzadeh G, Ghaffari MA, Bafandeh A, Hosseini SM, Ahmadi B. The relationship between-2548 G/A leptin gene polymorphism and risk of breast cancer and serum leptin levels in Ahvazian women. Iran J Cancer Prev. 2015; 8:100-108.

32. El-Hussiny MA, Atwa MA, Rashad WE, Shaheen DA, Elkady NM. Leptin receptor Q223R polymorphism in Egyptian female patients with breast cancer. Contemp Oncol. 2017; 21:42-47.

33. Hu X, Juneja SC, Maihle NJ, Cleary MP. Leptin--a growth factor in normal and malignant breast cells and for normal mammary gland development. J Natl Cancer Inst. 2002; 94:1704-1711.

34. Somasundar P, Yu AK, Vona-Davis L, McFadden DW. Differential effects of leptin on cancer in vitro. J Surg Res. 2003; 113:50-55.

35. Kasim NB, Huri HZ, Vethakkan SR, Ibrahim L, Abdullah BM. Genetic polymorphisms associated with overweight and obesity in uncontrolled Type 2 diabetes mellitus. Biomark Med. 2016; 10:403-415.

36. Lin HY, Shi H, Li CY, Chen QC, Huang TB, Liu PC, Lou LM. LEP and LEPR polymorphisms in non-Hodgkin lymphoma risk: a systematic review and pooled analysis. $\mathrm{J}$ BUON. 2015; 20:261-268.

37. He J, Xi B, Ruiter R, Shi TY, Zhu ML, Wang MY, Li QX, Zhou XY, Qiu LX, Wei QY. Association of LEP G2548A and LEPR Q223R polymorphisms with cancer susceptibility: evidence from a meta-analysis. PLoS One. 2013; 8:e75135.

38. Liu P, Shi H, Liu R, Yang Y, Yang Y, Huang C, Shu H, Gong J, Cai M. Lack of association between LEPR Q223R polymorphisms and cancer susceptibility: evidence from a meta-analysis. J BUON. 2014; 19:855-862.
39. Quinton ND, Lee AJ, Ross RJ, Eastell R, Blakemore AI. A single nucleotide polymorphism (SNP) in the leptin receptor is associated with $\mathrm{BMI}$, fat mass and leptin levels in postmenopausal Caucasian women. Hum Genet. 2001; 108:233-236.

40. Zhai Y, Zhao WH, Chen CM. [Verification on the cut-offs of waist circumference for defining central obesity in Chinese elderly and tall adults]. [Article in Chinese]. Zhonghua Liu Xing Bing Xue Za Zhi. 2010; 31:621-625.

41. Zhang X, Zhang S, Li Y, Detrano RC, Chen K, Li X, Zhao L, Benjamin EJ, Wu Y. Association of obesity and atrial fibrillation among middle-aged and elderly Chinese. Int J Obes. 2009; 33:1318-1325.

42. Yin J, Wang X, Wei J, Wang L, Shi Y, Zheng L, Tang W, Ding G, Liu C, Liu R, Chen S, Xu Z, Gu H. Interleukin 12B rs3212227 $\mathrm{T}>\mathrm{G}$ polymorphism was associated with an increased risk of gastric cardiac adenocarcinoma in a Chinese population. Dis Esophagus. 2015; 28:291-298.

43. Li Y, Sun Y, Wang Y, Sui H, Li Y, Dou Z, Gao Y, Xu H. Relationship between polymorphism of leptin receptor gene GIn223Arg and type 2 diabetes mellitus with chronic renal failure. Chin J Lab Diagn. 2015; 19:1670-1673.

44. Zhang S, Wang Y, Jiang H, Liu C, Sun B, Chen S, Kang M, Tang W. Peroxisome proliferator-activated receptor gamma rs $1801282 \mathrm{C}>\mathrm{G}$ polymorphism is associated with polycystic ovary syndrome susceptibility: a meta-analysis involving 7,069 subjects. Int J Clin Exp Med. 2015; 8:17418-17429.

45. Tang W, Wang Y, Jiang H, Liu P, Liu C, Gu H, Chen S, Kang M. Programmed death-1 (PD-1) rs2227981 C > T polymorphism is associated with cancer susceptibility: a meta-analysis. Int J Clin Exp Med. 2015; 8:22278-22285.

46. Qiu H, Cheng C, Wang Y, Kang M, Tang W, Chen S, $\mathrm{Gu}$ H, Liu C, Chen Y. Investigation of cyclin D1 rs9344 $\mathrm{G}>\mathrm{A}$ polymorphism in colorectal cancer: a meta-analysis involving 13,642 subjects. Onco Targets Ther. 2016; 9:6641-6650.

47. Tang W, Qiu H, Ding H, Sun B, Wang L, Yin J, Gu H. Association between the STK15 F31I polymorphism and cancer susceptibility: a meta-analysis involving 43,626 subjects. PLoS One. 2013; 8:e82790.

48. Tang W, Qiu H, Jiang H, Sun B, Wang L, Yin J, Gu H. Lack of association between cytotoxic T-lymphocyte antigen 4 (CTLA-4) -1722T/C (rs733618) polymorphism and cancer risk: from a case-control study to a meta-analysis. PLoS One. 2014; 9:e94039.

49. Tang W, Wang Y, Chen S, Lin J, Chen B, Yu S, Chen Y, $\mathrm{Gu} \mathrm{H}$, Kang M. Investigation of cytotoxic T-lymphocyte antigen 4 polymorphisms in gastric cardia adenocarcinoma. Scand J Immunol. 2016; 83:212-218. 